\section{Acknowledgement}

Authors are grateful to Pr. M. Matsubara (Dept. of Chem. Eng., Nagoya Univ.) for his valuable advices and discussions on the micromixing problemes, especially on the two-environment micromixing model.

\section{Nomenclature}

$a \quad=$ volume fraction of fluid $1\left(=C_{a} / C_{a 0}\right)$

$b \quad=$ volume fraction of fluid $2\left(=C_{b} / C_{b 0}\right)$

$C \quad=$ concentration

$D_{m} \quad=$ mass diffusivity

$d \quad=$ diameter of impeller

$=$ simultaneous frequency function of age and life expectation

$=$ intensity of segregation

$=$ degree of segregation based on age

$=$ reaction rate constant

$=$ power number

$==$ impeller speed

$=$ micromixing parameter

$=$ volumetric flow rate

$=$ micromixing parameter

$=$ Reynolds number

$=$ Schmidt number

$=$ segregation function

$=$ volume of tank reactor

$=$ age

$=$ turbulent kinetic energy dissipation rate per unitimass
$=$ life expectation

[sec]

$=$ microscale of turbulence

$=$ kinematic viscosity

$=$ density of reactant solution

$=$ mean residence time

$\phi_{L} \quad=$ life expectation distribution

〈Subscripts〉

$$
\begin{aligned}
& a \quad=\text { reactant } A \\
& b \quad=\text { reactant } B \\
& E \quad=\text { entering environment } \\
& e \quad=\text { exit } \\
& L=\text { leaving environment } \\
& 0 \quad=\text { time zero or initial time } \\
& 1 \text { = fluid } 1 \\
& 2=\text { fluid } 2
\end{aligned}
$$

\section{Literature Cited}

1) Brodkey, R. S.: "Mixing-Theory and Practice", Vol. 1, Chap. 2, p. 83, Academic Press, New York (1966).

2) Costa, P. and C. Trevissol: Chem. Eng. Sci., 27, 2041 (1972).

3) Danckwerts, P. V.: App. Sci. Res., 3, 279 (1953).

4) Goto, S. and M. Matsubara: Chem. Eng. Sci., 30, 61 (1975).

5) Leshaw, S. A.: Ph. D. Thesis, Columbia Univ. (1967).

6) Nishimura, Y. and M. Matsubara: Chem. Eng. Sci., 25, 1785 (1970).

7) Rao, D. P. and L. L. Edwards: ibid., 28, 1179 (1973).

8) Rippin, D. W. T.: ibid., 22, 247 (1967).

[sec] 9) Takao, M., Y. Muramoto., H. Inoue and Y. Murakami: Preprint of the 38th Annual Meeting of The Soc. of Chem. Engrs., Japan, 1, 170 (1973).

\title{
COMBINED PROCESS OF REACTION, EXTRACTION AND DISTILLATION
}

\author{
SHIGEO GOTO* AND MASAKAZU MATSUBARA \\ Department of Chemical Engineering, Nagoya University, Nagoya, 464
}

\section{Introduction}

In conventional chemical plants, processes to which different functions, such as reaction, extraction, distillation are assigned used to be treated independently of others except in some limited cases, e.g. the combination of reaction and heat exchange. But, under certain conditions, many other combined processes in which two or more operations are simultaneously processed might be preferable to the successive combination of conventional unit operations. For ex-

Received November 21, 1975.

Presented in part at the 9th Autumn Meeting of The Soc. of Chem. Engrs., Japan, at Fukuoka, Oct., 1975. ample, in esterification, a reactive distillation ${ }^{1,2)}$ and an extractive reaction ${ }^{3)}$ were investigated to obtain conversions higher than that for chemical equilibrium. For separation of mixtures having a very low relative volatility, an extractive distillation is often employed to change the relative volatility by adding another solvent.

In this paper, some basic considerations are given for assuring the advantage of the combined process where the three operations of reaction, extraction and distillation are simultaneously carried out. This combined operation which is referred to as reactive extractive distillation, is compared with three other processes, that is, reactive distillation, extractive 
reaction and reaction without simultaneous separation, in view of the yield of desired intermediate of the complex reaction in a stirred vessel.

\section{Problem Formulation}

Let us consider a single stirred tank involving two liquid phases and one vapor phase, as shown in Fig. 1. Two insoluble solvents form a reactive phase in which the reaction proceeds, and an extractive phase in which the extraction of desired product takes place. One of the two liquid phases is a dispersed phase and the other a continuous phase. The concentrations in each phase are homogeneous and the system is isothermal. The reactive and extractive phases are heated in their respective feed lines and their vapors evolve. For the reactive phase, the ratio of the vapor evolution rate, $v_{1}$, to the liquid feed flow rate, $g_{1 f}$, is equal to $\left(1-q_{1}\right)$. Similarly, for the extractive phase, the ratio of $v_{2}$ to $g_{2 f}$ is equal to $\left(1-q_{2}\right)$.

The reaction considered is a combination of reversible and consecutive reactions which are of the first order, that is,

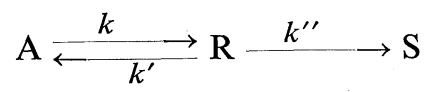

where $\mathrm{R}$ is the desired product and $\mathrm{S}$ the by-product.

The reaction rates in the reactive phase are

$$
\begin{gathered}
r_{A 1}=-k C_{A 1}+k^{\prime} C_{R 1}=-k C_{t 1} x_{A 1}+k^{\prime} C_{t 1} x_{R 1} \\
r_{R 1}=k C_{A 1}-\left(k^{\prime}+k^{\prime \prime}\right) C_{R 1}=k C_{t 1} x_{A 1}-\left(k^{\prime}+k^{\prime \prime}\right) C_{t 1} x_{R 1}
\end{gathered}
$$

It is assumed that no reactions occur in the vapor and extractive phases.

Mass balances of $\mathrm{A}$ and $\mathrm{R}$ around the stirred tank are

$$
\begin{gathered}
g_{1 f} x_{A 1, f}+g_{2 f} x_{A 2, f}=g_{1} x_{A 1}+g_{2} x_{A 2}+v y_{A}-r_{A 1} V_{1} \\
0=g_{1} x_{R 1}+g_{2} x_{R 2}+v y_{R}-r_{R 1} V_{1}
\end{gathered}
$$

where the concentrations of $\mathrm{R}$ at feed are zero.

Since the rate of mass transfer between each liquid and vapor phase is usually much higher than that of chemical reaction, vapor-liquid equilibrium is assumed and the equilibrium relationship is expressed in terms of the activity coefficient, $\gamma$. For the reactant, A, we have

$$
\begin{aligned}
P_{t} y_{A} & =\gamma_{A 1} P_{A} x_{A 1} \\
& =\gamma_{A 2} P_{A} x_{A 2}
\end{aligned}
$$

where $P_{t}$ is the total pressure and $P_{A}$ the vapor pressure of A. Rewriting these equations yields

$$
\begin{aligned}
& x_{A 2}=\left(\gamma_{A 1} / \gamma_{A 2}\right) x_{A 1}=K_{A} x_{A 1} \\
& y_{A}=\left(\gamma_{A 1} P_{A} / P_{t}\right) x_{A 1}=K_{A}^{*} x_{A 1}
\end{aligned}
$$

Similarly, for the intermediate, $\mathrm{R}$, we have

$$
\begin{aligned}
& x_{R 2}=\left(\gamma_{R 1} / \gamma_{R 2}\right) x_{R 1}=K_{R} x_{R 1} \\
& y_{R}=\left(\gamma_{R 1} P_{R} / P_{t}\right) x_{R 1}=K_{R}^{*} x_{R 1}
\end{aligned}
$$

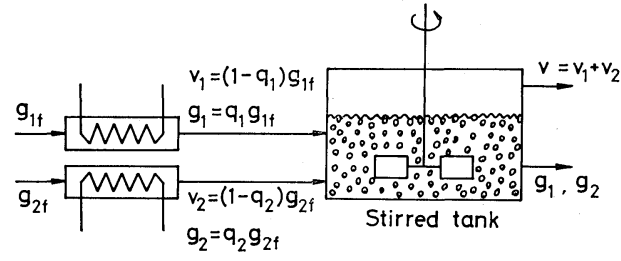

Fig. 1 Schematic of reactive-extractive distillation

For simplicity, activity coefficients are assumed to be independent of the concentrations and therefore $K_{A}$, $K_{A}^{*}, K_{R}$ and $K_{R}^{*}$ are constant. Since the mass transfer between two liquid phases is usually slower than that between vapor and liquid phases, it seems reasonable that the relationships of concentrations between two liquid phases are given by Eqs. (5) and (7) which depend only on the vapor-liquid equilibrium relationships. Therefore, the constants, $K_{A}$ and $K_{R}$ are generally different from the distribution coefficients of liquid-liquid equilibrium, $m_{A}$ and $m_{R}$.

By substituting Eqs. (1), (2), (5)-(8) into Eqs. (3) and (4) and by reducing to dimensionless equations, we have

$$
\begin{aligned}
\zeta x_{A 1, f}+(1-\zeta) x_{A 2, f} & =\left(\beta_{A}+\tau\right) x_{A 1}-\kappa_{R} \tau x_{R 1} \\
0 & =-\tau x_{A 1}+\left\{\beta_{R}+\left(\kappa_{R}+\kappa_{C}\right) \tau\right\} x_{R 1}
\end{aligned}
$$

where

$$
\begin{aligned}
\beta_{A} & =\left(g_{1}+g_{2} K_{A}+v K_{A}^{*}\right) /\left(g_{1 f}+g_{2 f}\right) \\
& =q_{1} \zeta+q_{2}(1-\zeta) K_{A}+\left\{\left(1-q_{1}\right) \zeta+\left(1-q_{2}\right)(1-\zeta)\right\} K_{A}^{*}
\end{aligned}
$$

$$
\begin{aligned}
\beta_{R} & =\left(g_{1}+g_{2} K_{R}+v K_{R}^{*}\right) /\left(g_{1 f}+g_{2 f}\right) \\
& =q_{1} \zeta+q_{2}(1-\zeta) K_{R}+\left\{\left(1-q_{1}\right) \zeta+\left(1-q_{2}\right)(1-\zeta)\right\} K_{R}^{*}
\end{aligned}
$$

$$
\begin{gathered}
\zeta=g_{1 f} /\left(g_{1 f}+g_{2 f}\right) \\
\tau=k C_{t 1} V_{1} /\left(g_{1 f}+g_{2 f}\right) \\
\kappa_{R}=k^{\prime} / k \\
\kappa_{C}=k^{\prime \prime} / k
\end{gathered}
$$

\section{Optimization}

The yield of the intermediate, $R$, is defined as the ratio of the resultant production rates of $R$ from vapor and liquid phases to the feed flow rates of $\mathrm{A}$;

$$
\eta=\left(g_{1} x_{R 1}+g_{2} x_{R 2}+v y_{R}\right) /\left(g_{1 f} x_{A 1, f}+g_{2 f} x_{A 2, f}\right)
$$

By using Eqs. (7), (8) and (12), we have

$$
\begin{aligned}
\eta & =\left(g_{1}+g_{2} K_{R}+v K_{R}^{*}\right) x_{R 1} /\left(g_{1 f} x_{A 1, f}+g_{2 f} x_{A 2, f}\right) \\
& =\beta_{R} x_{R 1} /\left\{\zeta x_{A 1, f}+(1-\zeta) x_{A 2, f}\right\}
\end{aligned}
$$

Further, by substituting $x_{R 1}$ derived from Eqs. (9) and (10), we obtain

$$
\eta=\beta_{R} \tau /\left[\kappa_{C} \tau^{2}+\left\{\beta_{A}\left(\kappa_{R}+\kappa_{C}\right)+\beta_{R}\right\} \tau+\beta_{A} \beta_{R}\right]
$$

Referring to Eqs. (11) and (12), we have four decision variables, $\tau, \zeta, q_{1}$ and $q_{2}$, and six parameters, $K_{A}, K_{R}, K_{A}^{*}, K_{R}^{*}, \kappa_{R}$ and $\kappa_{C}$ in Eq. (17). The value of 
Facet $\operatorname{DEFG}(\zeta=1)$ : Reactive distillation

Edge $B F\left(q_{1}=q_{2}=1\right)$ : Extractive reaction

Edge $\operatorname{EF}\left(\zeta=q_{1}=1\right)$ :Reaction

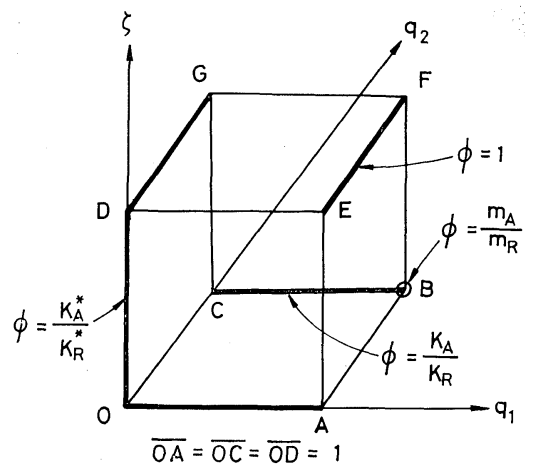

Fig. 2 The feasible region of $\zeta, q_{1}$ and $q_{2}$ for the reactive extractive distillation

each parameter is fixed if the reaction system, temperature and pressure are specified, whereas the value of each decision variable is adjustable by changing the operating conditions.

The value of $\tau$ which maximizes the value of $\eta$ can easily be determined as

$$
\tau=\sqrt{\beta_{A} \beta_{R} / \kappa_{C}}
$$

Substitution of Eq. (18) into Eq. (17) yields

$$
\eta=1 /\left\{\left(\kappa_{R}+\kappa_{C}\right) \phi+1+2 \sqrt{\kappa_{C}} \sqrt{\phi}\right\}
$$

where

$$
\begin{aligned}
\phi & =\beta_{A} / \beta_{R} \\
& =\frac{q_{1} \zeta+q_{2}(1-\zeta) K_{A}+\left\{\left(1-q_{1}\right) \zeta+\left(1-q_{2}\right)(1-\zeta)\right\} K_{A}^{*}}{q_{1} \zeta+q_{2}(1-\zeta) K_{R}+\left\{\left(1-q_{1}\right) \zeta+\left(1-q_{2}\right)(1-\zeta)\right\} K_{R}^{*}}
\end{aligned}
$$

In the case of extractive reaction for which $q_{1}=q_{2}=1$, $K_{A}$ and $K_{R}$ in Eq. (20) must be replaced by $m_{A}$ and $m_{R}$, respectively, as is pointed out previously. Therefore, Eq. (20) reduces to

$$
\phi=\left\{\zeta+(1-\zeta) m_{A}\right\} /\left\{\zeta+(1-\zeta) m_{R}\right\}
$$

Eq. (19) indicates that the maximization of $\eta$ reduces to the minimization of $\phi$ with respect to the remaining three variables, $\zeta, q_{1}$ and $q_{2}$. Since all these variables can be varied from zero to unity, the feasible region is the unit cube such as shown in Fig. 2. If we direct our attention to only one of these variables, we see that Eq. (20) or Eq. (21) has the form of

$$
\phi=(a \chi+b) /(c \chi+d)
$$

with respect to any one of them. It is clear that the $\phi$ in Eq. (20) or Eq. (21) is monotone increasing, monotone decreasing and constant according to $(a d-b c)$ being positive, negative and equal to zero, respectively. Therefore, the minimal value of $\phi$ must generally occur on the boundary of the cubic feasible region although under some special circumstances such as $K_{A} / K_{R}=K_{A}^{*} / K_{R}^{*}=1$ and $m_{A} / m_{R} \leq 1$ the same minimal value of $\phi$ is taken in the interior as well as on the boundary of the feasible region.

Now, consider the value of $\phi$ on the facet OABC in Fig. 2. Since $\zeta=0$ on this facet, Eqs. (20) and (21) reduce to

$$
\phi=\left\{q_{2} K_{A}+\left(1-q_{2}\right) K_{A}^{*}\right\} /\left\{q_{2} K_{R}+\left(1-q_{2}\right) K_{R}^{*}\right.
$$
and

$$
\phi=m_{A} / m_{R}
$$

respectively. The $\phi$ in Eq. (23) is independent of $q_{1}$ and the minimal value of $\phi$ will generally occur either on the edge $\mathrm{OA}$ or the edge $\mathrm{BC}$ because $\phi$ is monotonous with respect to $q_{2}$.

The $\phi$ takes the minimal value

$$
\phi=K_{A}^{*} / K_{R}^{*} \text { on the edge OA }\left(q_{2}=0\right)
$$

if $K_{A} / K_{R}>K_{A}^{*} / K_{R}^{*}$ and the minimal value

$$
\phi=K_{A} / K_{R} \text { on the edge BC }\left(q_{2}=1\right)
$$

if $K_{A} / K_{R}<K_{A}^{*} / K_{R}^{*}$.

In the special case of $K_{A}^{*} / K_{R}^{*}=K_{A} / K_{R}$, the $\phi$ is constant at every point on the facet OABC. In the discussion above, it is assumed that $m_{A} / m_{R}$ is larger than both $K_{A} / K_{R}$ and $K_{A}^{*} / K_{R}^{*}$. If this is not the case, the minimal value of $\phi$ on the facet OABC occurs at the vertex $\mathbf{B}$.

After the similar investigations about the remaining facets of the feasible cube, the candidates for the minimal value of $\phi$ are obtained as shown in Fig. 2.

\section{Conclusions}

It is evident from Fig. 2 that the minimal value of $\phi$ is the smallest of four values, $1, m_{A} / m_{R}, K_{A}^{*} / K_{R}^{*}$ and $K_{A} / K_{R}$ which can occur on the edge $\mathrm{EF}$, at the vertex $\mathrm{B}$, on the edges $\mathrm{OA}, \mathrm{OD}$ and $\mathrm{DG}$ and on the edge $\mathrm{BC}$, respectively. Therefore, the following conclusions are deduced:

(i) Since the edge EF corresponds to the reaction without simultaneous separation, any combined process is useless if the value 1 is the smallest.

(ii) Since the edge BF corresponds to the extractive reaction, the optimal combined process is the extractive reaction if the value $m_{A} / m_{R}$ is the smallest.

(iii) Since the facet DEFG corresponds to the reactive distillation, the optimal combined process is reactive distillation if the value $K_{A}^{*} / K_{R}^{*}$ is the smallest.

(iv) Reactive extractive distillation which includes all three phases in the stirred tank can be optimal on the edge $\mathrm{BC}$ (except the vertex B) only if the value $K_{A} / K_{R}$ is the smallest.

Since $\zeta=0$ and $q_{2}=1$ on the edge BC, there are no flow of reactive and vapor phases, and only the extractive liquid phase is flowing. But it is necessary to maintain three phases in the stirred tank because the reaction can take place only in the reactive phase, and in addition, the values of $K_{A}$ and $K_{R}$ come from 
vapor-liquid equilibrium. This requirement will be satisfied by the recirculation of reactive phase from a separator of the discharged liquid phases, and by using the closed stirred tank for maintaining the vapor phase. It will, however, be necessary in practice to feed make-up of reactive and vapor phases to the tank.

To improve the yield of the desired product, it is important to choose the two solvents having the value of $K_{A} / K_{R}, K_{A}^{*} / K_{R}^{*}$ or $m_{A} / m_{R}$ which is as small as possible.

\section{Nomenclature}

\begin{tabular}{|c|c|}
\hline$C$ & $=$ concentration \\
\hline$g$ & $=$ liquid flow rate \\
\hline$K_{A}$ & $=\gamma_{A 1} / \gamma_{A 2}$ \\
\hline$K_{R}$ & $=\gamma_{R 1} / \gamma_{R 2}$ \\
\hline$K_{A}^{*}$ & $=\gamma_{A 1} P_{A} / P_{t}$ \\
\hline$K_{R}^{*}$ & $=\gamma_{R 1} P_{A} / P_{t}$ \\
\hline$k, k^{\prime}, k^{\prime \prime}$ & $=$ rate constant of first order reaction \\
\hline$m$ & $=$ distribution coefficient \\
\hline$P$ & $=$ pressure \\
\hline $1-q$ & $\begin{aligned}= & \text { ratio of evolution rate of vapor to the } \\
& \text { liquid flow rate at feed }\end{aligned}$ \\
\hline$r$ & $=$ reaction rate \\
\hline$V$ & $=$ volume \\
\hline & $=$ vapor flow rate \\
\hline
\end{tabular}

$x \quad=$ mole fraction inli quid phase $\quad[-]$

$y \quad=$ mole fraction in vapor phase $\quad[-]$

$\beta_{A} \quad=$ defined by Eq. (11)

$\beta_{R} \quad=$ defined by Eq. (12) [-]

$\gamma \quad=$ activity coefficient $\quad[-]$

$\zeta \quad=g_{1 f} /\left(g_{1 f}+g_{2 f}\right)$

$\eta \quad=$ yield defined by Eq. (17) [-]

$\kappa_{R} \quad=k^{\prime} / k \quad$ [-]

$\kappa_{C} \quad=k^{\prime \prime} / k$ [-]

$\tau \quad=$ defined by Eq. (14) [-]

$\phi \quad=$ modulus defined by Eqs. (20) and (21) [-]

$\langle$ Subscripts〉

$A \quad=$ reactant, $\mathrm{A}$

$f \quad=$ feed

$R \quad=$ product, $\mathrm{R}$

$t \quad=$ total

$1 \quad=$ reactive phase

$2 \quad=$ extractive phase

Literature Cited

1) Nishi, Y.: Kagaku Kögaku, 35, 892 (1971).

2) Suzuki, I., H. Yagi, H. Komatsu and M. Hirata: J. Chem. Eng. Japan, 4, 26 (1971).

3) Washino, K., S. Goto and M. Matsubara: Kagaku Kōgaku, 38, 869 (1974).

\section{ABSTRACTS OF KAGAKU KOGAKU RONBUNSHU VOL. 2, NO. 4, JULY, 1976}

Light Scattering Aerosol Measurements for Absorbing Particles, Akira Kanagawa (Nagoya Univ.)

Kagaku Kogaku Ronbushu, 2, 325-330 (1976)

A discussion based on exact Mie theory calculations is given on light scattering aerosol measurements for absorbing aerosol particles. From the calculations of Mie's scattering light intensity functions for absorbing particles as a variable of imaginary part of refractive index, it has been found that the scattering light intensities vary abruptly with the variance of imaginary parts in the region from 0 to 0.2 of their value. Response calculations for the aerosol particle photocounter for particle sizing and the aerosol photometer for particle concentration measurement have been made for absorbing particles. As a result, it is pointed out that one should take into account the absorbing properties of aerosol particles on the light scattering aerosol measurement, particularly when the particle size is greater than $1 \mu \mathrm{m}$.

Size Determination of Submicron Particles by Owl-Type Photometer, Akira Kanagawa and Takeshi Takahashi (Nagoya Univ.) Kagaku Kogaku Ronbunshu, 2, 331-335 (1976)

A Owl-type photometer designed for measuring the particle size of monodispersed submicron particles was constructed and has been used experimentally for polystyrene latex hydrosols. The angular dependency of the ratio $R_{1}$ of the long-wavelength scattering light intensity vertically polarized to the short one are measured and compared with the theoretical relations calculated from Mie's scattering theory.

As a result, it was found that the pattern of the angular dependency of $R_{1}$ varies regularly with particle size, and this photometer seems to be capable of the size determination roughly of monodispersed particles in hydrosols.

Quite similar studies of the angular dependency of the polarization ratio of the scattering light were made also, and similar results were obtained.

Anisokinetic Sampling Errors for Aerosols, Hideto Yoshida, Hiroaki Masuda, Koichi Iinoya (Kyoto Univ.), Takeshi Ohsugi (Nihon Kogyo Co., Ltd.) and Shinichi Yuu (Kyushu Inst. of Tech.)

Kagaku Kogaku Ronbunshu, 2, 336-340 (1976)

To examine the error in measuring particle concentration due to anisokinetic sampling over a wide range of inertial parameters, 